\title{
EDUCACIÓN DIFERENCIAL EN LAS POLÍTICAS PÚBLICAS DEL DEPORTE, LA RECREACIÓN Y LA ACTIVIDAD FÍSICA EN COLOMBIA
}

\section{DIFFERENTIAL EDUCATION IN PUBLIC POLICIES OF SPORT, RECREATION AND PHYSICAL ACTIVITY IN COLOMBIA}

\author{
Luz Elena Gallo Cadavid ${ }^{1}$ \\ Zoraida Bolívar Cartagena ${ }^{2}$
}

\section{Resumen}

Este artículo surge de una investigación documental que tuvo por objetivo analizar la perspectiva diferencial en planes y políticas del sector del deporte, la recreación y la actividad física en Colombia, planteados para los periodos 2009-2016. Se hizo un estudio hermenéutico y el análisis de la información se centró en la temática de las diferencias y en la educación diferencial. La investigación muestra que las políticas públicas se mueven en la lógica binaria normal/anormal, inclusión/exclusión y prevalece la necesidad de ordenar y clasificar quiénes han sido los diferentes: los otros. La igualdad y la diferencia forman parte de los principales hitos discursivos en planes y políticas públicas. Como la educación parece más preocupada por la normalidad que por las diferencias hay que hacer resonar el lenguaje de la educación como una ética singular.

Palabras claves: pedagogía diferencial, cuerpo, educación física, diversidad, alteridad.

\section{Abstract}

This article arises from a documentary research which aimed to analyze the differential perspective in plans and policies for sport, recreation and physical activity in Colombia for a set period of 20092016. A hermeneutic study was done and the data analysis was focused on the issue of the differences and differential education. Research shows that public policies move in the binary logic normal/ abnormal, inclusion/exclusion and prevails the need to sort and classify Who have been the different ones?, the Others. Equality and difference are part of the main discursive milestones in plans and public policies. As education seems more concerned for normality than for differences, the language of Education must resonate as a unique ethic.

Keywords: differential pedagogy, body, physical education, diversity, alterity.

Fecha de recepción: 15 de octubre de 2014

Fecha de aprobación: 24 de marzo de 2015

Para citar este artículo:

Gallo, L.E. y Bolívar, Z. (2015). Educación diferencial en las políticas públicas del deporte, la recreación y la actividad física en Colombia. Lúdica Pedagógica, (21), 19-31.

\footnotetext{
1 Doctora en Educación. Docente titular de la Universidad de Antioquia (Colombia). Integrante del Grupo de Investigación Estudios en Corporal. Correo electrónico: luz.gallo@udea.edu.co

2 Licenciada en Filosofía. Universidad de Antioquia. Integrante del Semillero Estudios Corporales del Grupo de Investigación Estudios en Educación Corporal. Correo electrónico: zoraida.bolivar@udea.edu.co
} 


\section{INTRODUCCIÓN ${ }^{3}$}

"No se aprende la vida en el mar con ejercicios en un charco y, en cambio, un exceso de entrenamiento en un charco puede incapacitarnos para ser

Franz Kafka, Diar

Esta investigación pretende responder a los objetivos de la convocatoria realizada a través del trabajo conjunto entre Coldeportes y Colciencias sobre la educación diferencial en torno al deporte, la recreación y la actividad física en Colombia. ¿De qué manera algunos planes de desarrollo y decenales vinculan desde el deporte, la recreación y la actividad física el enfoque diferencial? ¿Se organizan grupos con enfoque poblacional para el desarrollo de algunos programas con el fin de dar cuenta del enfoque diferencial? ¿Se trata de organizar programas para cada grupo en condición de vulnerabilidad, género o etnia? ¿O hay otras interpretaciones del enfoque diferencial?

En los planes de desarrollo, en la normatividad referente al deporte, la recreación, la actividad física en Colombia y en el estudio Análisis de la implementación de políticas públicas en el deporte en el Valle del Cauca a partir de la Constitución de 1991 al 2011 (Loaiza, 2013), se le adjudican significados sociales y culturales, se dice que estas prácticas abren espacios para la inclusión social y para el ejercicio de los derechos por parte de los ciudadanos; generan espacios para la convivencia; impulsan reivindicaciones socioculturales; son indispensables para el pleno desarrollo de la personalidad; deben responder a las necesidades individuales y sociales; son excelentes alternativas para promover la inclusión y la igualdad, así como para mejorar el bienestar, la salud, la educación y la calidad de vida de las personas; el deporte es una herramienta fundamental para el desarrollo personal por su dimensión vivencial y experiencial; las niñas y las mujeres tienen acceso a oportunidades de liderazgo y pueden obtener experiencia en esta área; el deporte puede cambiar de manera positiva las normas que rigen los derechos de cada género. Las personas en situación de discapacidad se ven empoderadas por las oportunidades que representan los deportes.

Ahora bien, ¿estas prácticas deportivas, recreativas y de actividad física están ligadas a qué procesos de

3 El presente artículo forma parte de la investigación "Educación diferencial: un fundamento para las políticas públicas en deporte, recreación y actividad física", financiada por Colciencias-Coldeportes en la convocatoria 626-2013. diferenciación?, ¿el reconocimiento del otro pasa por el reconocimiento de las singularidades?, ¿a qué ideas de lo diferencial tienden estas prácticas? ¿Las actividades que se desarrollan desde el campo del deporte, la recreación y la actividad física han cambiado las representaciones, las imágenes, las miradas, los modos de narrar al otro? ¿Qué resultados han tenido los planes que se desarrollan con enfoque diferencial como estrategia para disminuir brechas de desigualdad en algunos de los sectores sociales, etarios y grupos étnicos mediante la promoción de la diversidad, la autonomía y el ejercicio de interculturalidad de ciudadanos a partir del reconocimiento de sus particularidades?

Hay un conjunto de significados relacionados alrededor de la idea de diferencia y hay todo un saber médico, social, político y educativo que diagnostica, clasifica, adjetiva y nombra las personas en un marco de definiciones. Con Michel Foucault (2007) se usa el término anormales para designar a los otros que se han clasificado con variadas tipologías como diferentes, deficientes, discapacitados, disfuncionales, con diversidades funcionales, con necesidades educativas especiales, deformes, rebeldes, extraños, entre otros (Guzmán, 2013; Planella y Pié, 2012; Gordillo 2007; Carpintero, 2009; De la Vega, 2010; Pérez de Lara, 2010; VeigaNieto, 2000). El juicio de anormalidad no es solo estadístico de distribución normal de fenómenos naturales, sociales y psicológicos (en los extremos de la campana de Gauss), es también un juicio moral dentro de lo que consideramos aceptable.

Y ¿qué significa lo normal?, para Nirje (citado por Sosa, 2009):

Significa un ritmo normal del día, levantarse de la cama a la hora que lo hace el promedio, salir para la escuela, al trabajo (no quedarse en la casa), hacer proyectos para el día, la semana. Ir a la escuela a un sitio, trabajar en otro, poder divertirse en otro sitio (no todo en la misma institución). Poder almorzar en la mesa (no en la cama). Tener "experiencias normales" de acuerdo con cada ciclo de vida. Poder tener diversas posibilidades de escoger, entre varios juegos o amigos. Que puedan enamorarse, vivir en un mundo de dos sexos, poder casarse o emparejarse... Tener derecho a un nivel de vida económico como el promedio de las personas, y tomar decisiones sobre en qué y cómo gastar su dinero (p. 66). 
En Colombia se encuentran estudios alusivos al deporte que se mueven entre la igualdad, la diferencia, la diversidad y la equidad (Pérez, 2013; Vásquez, 2012; Vélez, 2011; Loaiza, 2013; Benjumea, 2011; Hurtado, 2005; Alzate, Fragoso y Sarmiento, 2009), también se encuentran investigaciones sobre la educación especial en Colombia donde se resalta la necesidad de ampliar las relaciones entre la educación especial, la pedagogía crítica y las pedagogías diferenciadas (Yarza y Rodríguez, 2009; Yarza, 2007).

El trabajo sobre la diversidad en contextos educativos señalan la necesidad de un maestro capaz de trascender las miradas efímeras que se quedan en el déficit y la carencia, y que valide la diferencia como potencialidad en el reconocimiento del "sí mismo del otro, lo otro en relación con un nosotros" (Cajibioy y Sevilla, 2013). El estudio "Boccias como elemento de inclusión social", realizado con la participación de la Liga Vallecaucana de Parálisis Cerebral (Livapace) en el año 2011, concluye que los sujetos del estudio se perciben sin limitaciones aunque aún encuentren barreras en la sociedad (Jaramillo y Mejía, 2012).

Tradicionalmente, e incluso hoy en día, en diferentes regiones del mundo el concepto de inclusión educativa ha sido restringido a un grupo de estudiantes con necesidades especiales, como aquellos con discapacidades físicas y/o mentales y menores refugiados. Los enfoques y respuestas han sido esencialmente compensatorios $\mathrm{y} / \mathrm{o}$ correctivos, principalmente mediante el establecimiento de estructuras curriculares, programas de estudio y escuelas especiales diferenciadas. En muchos casos, una de las consecuencias más significativas de una estructura institucional y curricular diferenciada ha sido la segregación y el aislamiento, dentro del sistema educativo, de aquellos estudiantes considerados y a veces estigmatizados como con necesidades especiales (MEN, 2011).

La investigación El rastro de la diferencia, realizada sobre la práctica pedagógica en la educación especial en Colombia (Yarza y Rodríguez, 2009) dice que en 1923 la Asamblea Departamental de Antioquia definía a los anormales a partir de sus rasgos físicos, intelectuales y morales; los referían como atrasados, viciosos y torcidos, clasificaba a este tipo de población como inferiores. Las anormalidades intelectuales se clasificaban en idiotas, imbéciles y niños débiles. Según Yarza y Rodríguez (2009), "la educación física, la educación sensorial y las gimnásticas tenían como fin último normalizar, agenciar, encauzar para la producción económica y simbólica a una cantidad considerable de 'unidades' parcialmente improductivas" (p. 19).

Se dice que la forma de nombrar introduce la perspectiva de la diferencia (de lo diferente, de los diferentes, de las relaciones diferenciadas) en la reflexión educativa y se cree con ello que se habla de lo singular, de alguien que ha sido clasificado según parámetros de normalidad, es decir, por las normas que dictan aquello que debe ser normal orgánica, funcional y psicológicamente (Skliar y Pérez, 2014; Skliar y Bárcena, 2013; Dueñas, 2010; Irigaray y Vergara, 2011). Es evidente que el problema está en atender a los diferentes mediante actividades deportivas, recreativas y de actividad física; aquí la cuestión del otro se fundamenta en el discurso de lo patológico, lo deficiente, lo disfuncional, pareciera que la sospecha es el cuerpo normal, ese cuerpo que se objetualiza. Para Skliar (2011), "el problema es que la normalidad y el cuerpo normal han sido construidos para, al mismo tiempo, crear el problema del otro deficiente" (p. 135).

¿Por qué el desarrollo de la política pública en deporte, recreación y actividad física ha de partir de una concepción educativa de las diferencias? Se cree que educar "bien" deviene de la adopción crucial de un conocimiento acerca del otro, de la infancia, la juventud, ciertas comunidades o de ciertos sujetos en determinadas y particulares condiciones de existencia. Y se ha pensado que lo esencial de educar resulta en una práctica derivada directamente de ese conocimiento, sin embargo, esto es insuficiente si no se reconoce al género, la etnia, la identidad sexual, la situación de salud y de clase como construcciones sociales, culturales e históricas cambiantes.

¿Cómo pensar una educación de las diferencias hoy que se habla de una educación para todos? ¡Todos sin excepción!, aunque sabemos que no parece caber un cualquiera: cualquier niño, niña, cualquier joven, en fin, cualquier otro, con cualquier cuerpo, cualquier modo de aprender, cualquier posición social, cualquier sexualidad, cualquier raza, cualquier condición social. ¿Pero, quién es el otro? Con Skliar (2011), "todos somos, en cierto modo, otros o bien todos somos, en cierto modo, diferentes" (p. 83). Como el desafío de incluir a todos fue haciéndose cada vez más abstracto, este estudio pretende mostrar que nos hace falta un 
proyecto educativo en relación con las diferencias que ponga en tela de juicio más la normalidad que la anormalidad (Skliar, 2005). Desde estos planteamientos, ¿de quién o de quiénes resulta ser el argumento de la diferencia?

En estudios realizados por Sosa (2007) se muestran los cuerpos de los peros:

"Sí, pero el problema está en el traslado al campo de deportes, él va a un ritmo más lento que el resto" "Pero hicimos un acuerdo de convivencia diferente, para todo lo que sea diferente, es la actitud frente a la diferencia, la que tuvimos que cambiar"

"Pero a esta nena que la rechazan de todos lados, tiene un retraso madurativo importante, en ese momento no me había dado cuenta"

“¿Pero dónde está el límite? Con un problema neurológico, que le afectó la motricidad en piernas y a las maestras les da mucho trabajo y recomiendan otra escuela".

"Se lo quieren sacar de encima... pero ¿puedo yo obligar a un docente a aceptar todas las diferencias?" Pero no estamos preparados para "esto".

"No me pueden obligar a atender a un cuadripléjico, estamos hablando de un motor, yo igual lo integro, aunque sea de arquero, pero lo integro" (p. 101, 102).

Estudios de la educación diferenciada estilan en enseñanzas diferenciadas e intereses acerca de comparar entre una educación diferenciada y la no diferenciada (Salomone, 2007). Respecto a los discursos de la educación inclusiva, Sánchez, Díaz, Sanhueza y Friz (2008) manifiestan que "uno de los pilares o requerimientos para que la inclusión sea efectiva es la formación del profesorado para atender las características heterogéneas del alumnado" (p. 170); además la inclusión ha sido tradicionalmente una acción directa de la denominada educación especial que hoy pasa a denominarse atención a la diversidad. También hay que revisar cuál es la pregunta de la inclusión educativa cuando la escuela dice "no estamos preparados", o el profesor menciona "no estoy preparado para atender a ese niño, a esa niña o a ese joven". 0 como señala una profesora de educación física: "nosotros tratamos de asignarles algunas tareas pero en realidad no es la calidad que se merece ese niño con esa necesidad educativa especial... a esos niños uno les pone cualquier cosita o los deja por ahí". También hay un reclamo por pensar de otras maneras la educación diferencial o pedagogías de las diferencias (Pié, 2014; Dussel, 2013; Ripamonti,
2011; Mèlich, 2010; Skliar y Larrosa, 2009; Pérez de Lara, 2009; Skliar, 2008, 2002; Skliar y Téllez, 2008; Ribetto, 2005; Duschatzky y Skliar, 2001; Frigerio y Diker, 2010).

Hasta ahora, la educación no problematiza lo normal, la normalización y los parámetros se instalan en la repetición, lo homogéneo, la mismidad, la identidad, lo que debe ser de acuerdo con los modelos que instala lo normal al cuerpo, la lengua, el aprendizaje, la enseñanza, el comportamiento. La educación no se preocupa por las diferencias sino por los diferentes, los extraños por categorizar y por identificar, ¿quiénes son los diferentes?, los otros que han ocupado espacios de alteridad en virtud de etnia, raza, identidad sexual, clase social o simultáneamente, negro, indígena, loco, discapacitado, desplazado, inmigrante, mujer, niño(a), tercera edad, ateo, etc. No parece haber consenso alrededor de las maneras de entender lo diferencial porque pareciera que la preocupación radica en los diferentes.

Para Skliar y Téllez (2008):

Los "diferentes" obedecen a una construcción, una invención, son un reflejo de un largo proceso que podríamos llamar de "diferencialismo'; esto es, una actitud -sin dudas racista- de separación y de disminución de algunos trazos, de algunas marcas, de algunas identidades con relación a la vasta generalidad de las diferencias. Las diferencias no pueden ser presentadas ni descriptas en términos de mejor o peor, bien o mal, superior o inferior, positivas o negativas, etcétera. Son, simplemente, diferencias. Pero el hecho de traducir algunas de ellas como "diferentes" y ya no como diferencias vuelve a posicionar estas marcas como contrarias, como opuestas y negativas a la idea de "norma"; de lo "normal" y, entonces, de lo "correcto", de lo "positivo"; de lo "mejor", etc. (pp. 9 -10).

La idea de la diferencia y lo diferencial no trata solamente de una razón jurídica sino de una razón ética; según Skliar (2008), "en el pensamiento de la diferencia no existen sujetos diferentes" (p. 14). Si hablamos de las diferencias de cuerpo, todos los cuerpos forman parte de ella; si hablamos de las diferencias de aprendizaje, todos los modos de aprender caben en ella; si hablamos de las diferencias de lenguaje, todos los modos de producción y comprensión están allí (Skliar, 2008). Una mirada ética hacia las diferencias ha de reconocer que la vida se da en múltiples formas, que los cuerpos forman parte de la diferencia, y no de los 
sujetos que se señalan, diagnostican o clasifican como diferentes. Lo diferencial ya no apunta hacia el otro o lo rotula como diferente, sino que pasa a ser una mirada puesta en un nosotros, en aquello que pasa entre nosotros e incluso en una idea potente para la educación como es el estar juntos, porque las diferencias nos permiten estar juntos y pueden ser una clave de transformación política y educativa.

\section{MÉTODO}

Esta investigación se inscribe en un enfoque cualitativo con un alcance descriptivo y es de tipo documental. Se hizo un análisis sistemático de la información sobre planes y políticas del sector del deporte, la recreación y la actividad física en Colombia planteados para los periodos 2009-2016 principalmente en Bogotá y Antioquia. El criterio de selección del periodo de análisis acata dos intereses, el primero, del "Plan decenal del deporte, la recreación, la educación física y la actividad física para el desarrollo humano, la convivencia y la paz 2009-2019", en el cual se establecen los lineamientos de política pública para deporte, la recreación y la actividad física para Colombia. El segundo interés obedece a los periodos de los planes de desarrollo entre 2012-2016 en los cuales se pone de manifiesto el criterio de enfoque diferencial para la actividad física, la recreación y el deporte.

El análisis de la información se centró en la temática de las diferencias y en la educación diferencial y se hizo un estudio hermenéutico a partir de las fases propuestas por Danner (citado por Krüger, 1999) para la interpretación de textos: preparatoria, inmanente y coordinada. En la fase de interpretación preparatoria se identificaron textos relativos a los planes de desarrollo, políticas públicas del sector del deporte, la recreación y la actividad física, leyes, normas, decretos del contexto nacional y estudios nacionales realizados sobre planes y programas en relación con el enfoque diferencial. En la fase de interpretación inmanente se hizo un análisis categorial y se identificaron patrones y tendencias de lo diferencial en los planes y políticas del sector del deporte, la recreación y la actividad física. En la fase de interpretación coordinada se observó la concepción de lo diferencial en perspectiva educativa y se hizo una lectura cruzada de los textos.
El análisis de la información se realizó con el programa Atlas.ti versión 6.0 que permitió organizar, clasificar, codificar y categorizar los textos, lo cual facilitó su relación. Se realizó la triangulación de los resultados de manera múltiple con dos puntos de referencia, con los actores o investigadores del estudio y con los datos.

\section{RESULTADOS}

“Desde luego sería mucho más fácil, mucho más cómodo y mucho más profesional (pero sin nada de amorosidad) si comprendiésemos al otro

sólo como una temática (el otro se convierte en un tema, siempre es un tema: así, por ejemplo, no hay niños ni niñas sino 'infancia', no hay sordos y sordas sino 'sordera'; no hay pobres sino 'pobreza'; 'indigencia', 'clases sociales,' 'clases bajas', etc. [...] tal vez para ratificar el abandono del otro y la inmunidad al otro". Carlos Skliar y Magaldy Téllez (2008).

Visibilización del enfoque diferencial en políticas y planes del deporte, la actividad física y la recreación en Colombia

$\mathrm{Al}$ analizar la perspectiva diferencial en planes y políticas públicas del sector deporte, la recreación y la actividad física en Colombia encontramos que en ellos subyace el problema de la igualdad y la diferencia. Un primer elemento a recordar es que, a partir del siglo XVIII, los fundamentos de la modernidad hicieron del principio de igualdad uno de sus supuestos más indicativos y la igualdad está en el imaginario ideológico y, desde la Revolución francesa, ha estado asociada con derechos.

La temática de diferencia y la igualdad se fundamenta en los movimientos sociales, las corrientes postcoloniales y feministas, los estudios culturales, la filosofía de la diferencia, que, desde una posición crítica de los postulados de la modernidad, cuestionan los criterios de naturalismo, neutralidad, objetividad, universalidad y revelan posibilidades para pensar la pluralidad, la diversidad, la multiplicidad y la alteridad.

Aunque en los planes y políticas se utiliza la expresión reconocimiento de las diferencias, también encontramos las expresiones reconocimiento de la diversidad y el reconocimiento a la igualdad, en ambos casos, se trata de teorías de transformación social y de resistencia, con una particular preocupación por las subjetividades y por los discursos que han sido invisibilizados. En cada una de ellas existen, a su vez, múltiples y diversos enfoques, por lo cual no pueden ser considerados únicos ni homogéneos. 
Cuadro 1. Análisis del enfoque diferencial en políticas y planes del deporte, la actividad física y la recreación en Colombia

\begin{tabular}{|c|c|}
\hline Documento & Fragmentos referidos al deporte, la recreación y la actividad física \\
\hline $\begin{array}{l}\text { Constitución Política de Colombia. } \\
\text { Artículo } 52\end{array}$ & $\begin{array}{l}\text { Reconoce el derecho de todas las personas a la recreación, a la práctica del deporte y al } \\
\text { aprovechamiento del tiempo libre. }\end{array}$ \\
\hline $\begin{array}{l}\text { Carta Internacional de la Educa- } \\
\text { ción Física y el Deporte, procla- } \\
\text { mada en } 1978 \text { (Unesco) }\end{array}$ & La práctica de la educación física y el deporte es un derecho fundamental para todos. \\
\hline $\begin{array}{l}\text { Plan Nacional de Desarrollo } \\
2010-2014 . \\
\text { Prosperidad para todos }\end{array}$ & $\begin{array}{l}\text {-Incrementar y mejorar la oferta y facilitar el acceso a programas de recreación, deporte y } \\
\text { cultura que permitan el adecuado manejo del tiempo libre, teniendo en cuenta las diferen- } \\
\text { cias culturales y los menores en condición de discapacidad. } \\
\text {-El deporte, la recreación y la actividad física representan una valiosa estrategia para el } \\
\text { bienestar, la salud, la educación y las políticas de inclusión por su contribución a los fines } \\
\text { sociales del Estado. } \\
\text {-Las políticas de recreación y deporte se desarrollarán, contemplando en lo pertinente, } \\
\text { enfoques diferenciales que permitan atender las disparidades poblacionales y regionales. } \\
\text {-Definir lineamientos pedagógicos para una educación diferencial. } \\
\text {-Las políticas del deporte están señaladas en el Capítulo IV como "Igualdad de } \\
\text { oportunidades". }\end{array}$ \\
\hline $\begin{array}{l}\text { "Plan Decenal del deporte, la } \\
\text { recreación, la educación física y la } \\
\text { actividad física, para el desarrollo } \\
\text { humano, la convivencia y la paz. } \\
\text { 2009-2019". } \\
\text { Instituto Colombiano del Deporte } \\
\text { Coldeportes }\end{array}$ & $\begin{array}{l}\text {-El Plan reconoce al deporte, la recreación, la educación física y la actividad física como ins- } \\
\text { trumentos para el desarrollo nacional, por cuanto son medios de expresión de diversidad } \\
\text { y multiculturalidad que demandan una comprensión multicausal e intervenciones diferen- } \\
\text { ciadas de acuerdo con las particulares necesidades de la población colombiana. } \\
\text {-Deberá formularse una política diferenciada para la infraestructura deportiva. } \\
\text {-Colombia necesita proyectar al sector como estrategia para construir oportunidades de } \\
\text { participación social, garantizando su acceso y práctica en condiciones dignas para la niñez, } \\
\text { la juventud, los adultos, las personas en situación vulnerable o de discapacidad, las mujeres } \\
\text { y los adultos mayores. } \\
\text {-Las poblaciones vulnerables, como los adultos mayores o la población con discapacidad, } \\
\text { se deben incluir más fácilmente a través de una oferta de recreación que paulatinamente } \\
\text { contribuya a su expresión autónoma no dirigida externamente. } \\
\text {-Integra las normas referidas al reconocimiento de los derechos para la población con dis- } \\
\text { capacidad y la creación del Comité Paralímpico. } \\
\text {-Se establecen lineamientos para la práctica del deporte, la recreación y la actividad física } \\
\text { de la población en situación de discapacidad. } \\
\text {-Promoción de alianzas entre diversos sectores con el deporte asociado en sus modalidades } \\
\text { convencionales y de inclusión de la discapacidad. }\end{array}$ \\
\hline
\end{tabular}




\begin{tabular}{|c|c|}
\hline Doc & Fragmentos referidos al deporte, la recreación y la actividad física \\
\hline $\begin{array}{l}\text { Plan de Desarrollo de Antioquia } \\
2012-2015 . \\
\text { "Antioquia la más educada” } \\
\text { Indeportes Antioquia }\end{array}$ & $\begin{array}{l}\text { En la línea } 3 \text { "Antioquia es segura y previene la violencia } \\
\text { " se encuentra el programa "Construyendo ciudadanía prevenimos la violencia y la reinci- } \\
\text { dencia”, y como proyecto: juegos municipales, subregionales, departamentales y fronteri- } \\
\text { zos para el encuentro y la convivencia. } \\
\text { En la línea } 4 \text { "Inclusión social } \\
\text { ", se desarrollan como proyectos: } 1 \text {. Actividad física para la salud ("Por su salud, muévase } \\
\text { pues"); } 2 \text {. Deporte y recreación para la inclusión y la interculturalidad. } \\
\text { En el programa “Cerrando brechas" se encuentra el proyecto "Deporte y recreación para la } \\
\text { inclusión y la interculturalidad para el encuentro ciudadano, la inclusión y la generación de } \\
\text { convivencia". } \\
\text { Los centros recreodeportivos para personas en situación de discapacidad. }\end{array}$ \\
\hline $\begin{array}{l}\text { Plan de Desarrollo } \\
\text { 2012-2015. } \\
\text { “Medellín un hogar para la vida” }\end{array}$ & $\begin{array}{l}\text { Componente 4. Deporte y recreación. } \\
\text { Entre los programas que contiene este plan para el sector del deporte, la recreación y la } \\
\text { actividad física se encuentran: 1. Jóvenes por la vida, una estrategia deportiva y recreativa } \\
\text { 2. Medellín feliz 3. Ciudad viva: administración, construcción, adecuación y mantenimiento } \\
\text { de escenarios deportivos y recreativos } 4 \text {. Desarrollo deportivo y recreativo, base para la } \\
\text { vida. } \\
\text { Programas deportivos en el sector educativo, asociado y comunitario. Escuelas Populares } \\
\text { del Deporte y Adrenalina. } \\
\text { Programas recreativos: Ludotekas, Mientras volvemos a casa y Recreando. } \\
\text { Programas de Estilos de vida saludable: promoción de Estilos de vida saludable, fortaleci- } \\
\text { miento de la Actividad física saludable, Deporte sin límites y ciclovías. }\end{array}$ \\
\hline $\begin{array}{l}\text { Política Pública de Deporte, } \\
\text { Recreación y Actividad Física para } \\
\text { Bogotá } \\
\text { 2009-2019. } \\
\text { "Bogotá más Activa". }\end{array}$ & $\begin{array}{l}\text { Componentes de esta política pública: } 1 \text {. Aumentar la participación } 2 \text {. Fortalecer la institu- } \\
\text { cionalidad 3. Ampliar la formación } 4 \text {. Optimizar la información y la comunicación } 5 \text {. Mejo- } \\
\text { rar los parques, escenarios y entornos. } \\
\text { Deben ofrecerse oportunidades especiales a los jóvenes, niños y niñas de edad preescolar, } \\
\text { personas de edad y a quienes tienen algún tipo de discapacidad. }\end{array}$ \\
\hline
\end{tabular}




\begin{tabular}{|l|}
\hline Documento \\
\hline Plan de Desarrollo \\
2012-2016 \\
"Bogotá Humana" \\
Instituto Distrital de Recreación y \\
Deporte (IDRD)
\end{tabular}

Fragmentos referidos al deporte, la recreación y la actividad física

Una ciudad que reduce la segregación y la discriminación: el ser humano en el centro.

Artículo 6. Estrategia 10. Reconocer la dimensión específica de la cultura, el arte, la actividad física, la recreación y el deporte bajo un criterio de enfoque diferencial y de respeto por la diversidad existente en razón a la edad, el género, la pertenencia cultural y étnica, la orientación sexual, la identidad de género, la apariencia y la discapacidad.

Artículo 9. Construcción de saberes. Educación incluyente, Diversa y de calidad para disfrutar y aprender.

Artículo 10. Programa Bogotá Humana con igualdad de oportunidades y equidad de género.

Artículo 11. Programa lucha contra distintos tipos de discriminación y violencias por condición, situación, identidad, diferencia, diversidad o etapa del ciclo vital, su propósito es transformar condiciones, actitudes y comportamientos que generan percepción de discriminación para disminuir la exclusión, la segregación y la marginación de pueblos afrodescendientes, palenqueros raizales, indígenas y rom, poblaciones y grupos poblacionales minoritarios tradicionalmente vulnerados.

Artículo 14. Programa ejercicio de las libertades culturales y deportivas. Este promueve el reconocimiento y el despliegue de las prácticas artísticas, culturales, recreativas y deportivas de las diferentes poblaciones, disminuyendo las barreras económicas, sociales, territoriales y mediando ante las barreras culturales que limitan su libre ejercicio y visibilización.

Programas: Muévete Bogotá, Ciclovía, Recreovía, Programas recreativos para personas mayores y para personas con limitación, Recreación para la infancia y para la juventud, Recreación comunitaria.

Programas deportivos.

El Decreto 1930 del 11 septiembre de 2013 de la República de Colombia, por el cual se adopta la Política Pública Nacional de Equidad de Género y se crea una Comisión Intersectorial para su implementación

El lineamiento 10 de la Política Pública Nacional de Equidad de Género para las Mujeres contempla el "fomento del deporte y recreación con enfoque diferencial y del uso creativo del tiempo libre y los estilos de vida saludables" y en concordancia con el Documento Conpes Social es necesario proponer estrategias y acciones para la Comisión intersectorial de Coordinación de la Política Pública, en este desde Coldeportes junto con la Alta Consejería Presidencial para la equidad de las mujeres, Ministerios de Salud y Educación.

\section{Términos relacionados con lo diferencial}

Derecho, atención diferencial, análisis diferenciales de contexto y de diversidad, atención a las necesidades, características y particularidades especiales de los sujetos, igualdad de oportunidades, diversidad, multiculturalidad, inclusión social, no discriminación, diversidad, ciudadanía, interculturalidad, pluralismo, discapacidad, diversidad funcional, equidad, atención a personas en situación de vulnerabilidad, atención a personas según ámbitos geográficos y/o poblacionales, capacidades y oportunidades incluyentes, capacidades diversas, valoración de lo común

Aunque no hay dos iguales, la aspiración a la igualdad parece estar presente en algunas políticas públicas del deporte, la recreación y la actividad física, así como en algunos enfoques de género y de etnia. El Programa de las Naciones Unidas para el Desarrollo (PNUD) señala que en el campo de las políticas públicas, el tema de la igualdad está ligado a diversos hechos de los cuales conviene destacar: la estrecha conexión entre el principio de igualdad y el principio de no discriminación; la articulación con el principio de equidad como 
elemento fundamental del debate; las elaboraciones a que ha dado lugar la diferencia entre la llamada igualdad de hecho o de facto y la igualdad de derecho.

Una lectura crítica que se hace a la igualdad es su controvertible valor, es un ideal que habla de un mundo que no es, de aquello a lo que se aspira, de grandes principios, de fundamentos del derecho, etc.; sin embargo, al parecer pierde el contacto con las personas y con las relaciones concretas. Y cuando se asocia con lo concreto, lo hace desde la norma que exige tratar o pedir a todos lo mismo; por ejemplo, algunas organizaciones abogan por políticas de igualdad. Cuando se hace referencia a la igualdad operamos en un reduccionismo porque se habla de aquello que iguala, o que se iguala, o que se presenta como igual, dejando de lado las diferencias. Entonces, igual se equipara con lo idéntico, con lo mismo y con homogeneidad.

¿Igualdad de qué?, ¿iguales a quién? Ambas preguntas remiten a un referente idealizado, según el cual unos tienen que moverse en la dirección de los atributos que los otros manifiestan. Las mujeres, hacia los hombres; las gentes llamadas de color, hacia las llamadas blancas; quienes tienen discapacidades, hacia los ¿normales?; la expresión de igualdad ignora o suprime todo aquello que no queda igualado, esto es, identificado en lo mismo. No hay que identificar cualquier diferencia con una desigualdad, deficiencia o anormalidad. De lo que se trata es de partir de otro lugar que no esté atrapado en este juego de oposiciones entre igualdad/ homogeneidad/mismidad desigualdad/deficiencia/ anormalidad, para poder ver algo más conectado con la experiencia, con lo concreto y con lo singular (Contreras, 2013).

Si bien la denominada etapa post Beijing está caracterizada por la emergencia de importantes categorías de análisis y aplicación de las políticas públicas que suponen la igualdad de oportunidades, aunque se avanza hacia la igualdad, al mismo tiempo, emerge el valor de la diversidad. Los análisis y propuestas del feminismo, discursos poscoloniales y algunos de los recientes desarrollos teóricos sobre masculinidades, aportan a la tipificación de políticas públicas basadas tanto en la igualdad como en las diferencias.

El enfoque diferencial en las políticas del deporte, la recreación y la actividad física reconoce que todo el accionar público está anclado en un sistema de derechos y deberes establecidos por un marco normativo nacional e internacional, mediante el cual se busca "potenciar la capacidad de acción efectiva de la población, especialmente de los grupos más marginados para participar en la formulación de políticas y hacer responsables a los que tienen la obligación de actuar" (ONU, 2006).

\section{Para Aldana et al. (2011):}

El enfoque diferencial es una estrategia que tiene como propósito disminuir las brechas de desigualdad existentes en algunos de los sectores sociales, etarios y grupos étnicos mediante la promoción de la diversidad, la autonomía y el ejercicio de interculturalidad de las ciudadanas y los ciudadanos, a partir del reconocimiento de sus particularidades según lo establecido por el Derecho Internacional, el cual reconoce que ciertos grupos de personas tienen necesidades de protección diferenciales a de sus situaciones específicas, y en algunos casos, de su vulnerabilidad manifiesta o de las inequidades estructurales y se sustenta en criterios de carácter étnico, etario y social para definir los grupos poblacionales a los cuales va dirigido el enfoque ( $p$. 6).

El análisis también pone en evidencia el interés que tienen las políticas y planes para promover la inclusión, la equidad y la participación de los ciudadanos en el deporte, la recreación y la actividad física. A su vez, hay una tendencia hacia lo educativo aunque se cree que educar bien deviene de la adopción crucial de un conocimiento acerca de otro, de la infancia, o de la juventud, o de ciertas comunidades, o de ciertos sujetos en determinadas y particulares condiciones de existencia. Y se ha pensado que lo esencial de educar resulta en una práctica derivada directamente de ese conocimiento; sin embargo, esto es insuficiente si no se reconoce al género, la etnia, la identidad sexual, la situación de salud y de clase como construcciones sociales, culturales e históricas cambiantes (a partir de las cuales se definen roles, actividades, espacios, valores y el poder que se detenta por unos y otros), porque esto ha permitido romper con concepciones biologicistas y esencialistas que legitiman órdenes socioeconómicos y políticos establecidos.

Desde estos planteamientos, la indagación por la Educación diferencial en las políticas públicas del deporte, la recreación y la actividad física aún se limita a pautas de enseñanza fundamentadas en la funcionalidad, 
el establecimiento de grupos homogéneos y en procesos clasificatorios lo cual escasea la diversidad como tópico crucial de toda política pública. Lo diferencial no se reduce a establecer programas para los diferentes o grupos con enfoque poblacional en condición de vulnerabilidad, género o etnia que hay que atender, capacitar, dotar, normalizar, perfeccionar, etc., ni apunta con lo que falta, ni con la ausencia, ni con lo que supone desvío, ni anormalidad.

\section{DISCUSIÓN Y CONCLUSIONES}

Este estudio pone en evidencia que una política pública en deporte, recreación y actividad física debe articularse no solo con el sector educativo, sino con una perspectiva educativa; en el campo discursivo proliferan los llamados a hacer de la educación el eje fundamental de las reformas sociales, culturales y políticas.

Esta investigación pone de manifiesto que la educación diferencial es un tópico fundamental del debate actual en cuyas coordenadas se habla por ejemplo de la diversidad cultural, la ciudadanía, los derechos humanos, la pluralidad, el reconocimiento del otro, la inclusión, etc. Sin embargo, en las políticas públicas del deporte, la actividad física y la recreación la noción de la diferencia y lo diferencial se confunde con discapacidad, inclusión/exclusión, normalidad/anormalidad, entre otros. Esta habitual confusión se origina justo cuando las diferencias se hacen presentes y son nombradas o se utilizan para organizar programas, pues pareciera que en el acto mismo de enunciar la diferencia, sobreviene una derivación hacia otra pronunciación de los diferentes como los otros, el otro que, se dice, ocupa los espacios de la alteridad.

Además, las políticas insisten en la inclusión, la integración, la diversidad, la participación como componentes centrales de los planes y programas; ¿lo que está en juego en los planes y políticas públicas es una nueva retórica? Para el desarrollo de una cultura de la diversidad es necesario ampliar la concepción de educación porque esta no se lleva a acabo solo en la institución escuela; una política pública ha de tener una concepción educativa colectiva que trascienda la transmisión y la información, la teoría separada de la práctica, tiene el desafío de gestionar y desarrollar proyectos educativos útiles, contextualizados a las problemáticas sociales y culturales. La población denominada diferencial es portadora de valores, identidades y sentidos. Sin embargo, si las políticas públicas no tienen una concepción amplia de la educación diferencial que gestione, promueva y fomente la cultura desde, por y para la diversidad, los ciudadanos y ciudadanas no tendrán acceso a prácticas culturales como deporte, recreación, literatura, cine, música, teatro, a la memoria colectiva ni al patrimonio. No tendrían acceso a producir, ni a disfrutar de la cultura. No accederán en igualdad de condiciones a múltiples bienes culturales que les confrontan con sus propias experiencias, que amplían la sensibilidad cultural. Porque no es suficiente con hacer reportajes de prácticas culturales con las poblaciones haciendo referencia a indicadores que miden el número de asistentes hacia una actividad, desconociendo los sentidos, los significados, las influencias, los imaginarios, así como las maneras como interpretan su existencia y experiencia diferenciales. Todo ello debido a los estereotipos promovidos socialmente y que relegan a la población diferencial y la marginan del contexto cultural general porque la diversidad cultural hace referencia a la coexistencia de las diferencias.

Este estudio demuestra, al igual que algunos autores de la Flacso ${ }^{4}$, que se viene confundiendo la noción de las diferencias y lo diferencial con los diferentes, con los cuerpos que son pensados como diferentes, extraños, los otros. El hecho de nombrar a los diferentes nos pone a pensar en términos de oposiciones respecto a la idea de lo mismo porque no todas las personas tenemos la misma lengua, cultura, raza, religión, cuerpo, tal vez, lo importante no es la obsesión por el otro sino la cuestión de la alteridad.

Por ello, hay que pensar los modos en que se produce lo educativo cuando se trabaja con enfoque diferencial en los programas del deporte, la actividad física y la recreación. Esta investigación considera que es apoyar el desarrollo de políticas públicas en deporte, recreación y actividad física a partir de una concepción educativa de las diferencias o pedagogías de las diferencias o pedagogía de la alteridad, lo cual implica alterar ciertos lenguajes con los que pensamos, decimos y actuamos en la educación. Una educación que signifique menos educar a otro y más educar con el otro, un estar juntos, en donde va a ser más importante el sentido de

\footnotetext{
4 Facultad Latinoamericana de Ciencias Sociales, Sede Académica Argentina.
} 
humanidad, el favorecimiento de las singularidades y el reconocimiento de todo ser humano como un sujeto de capacidades.

Educar también exige un gesto inicial de igualdad como punto de partida no como punto de llegada; una igualdad que no es homogeneidad, normalidad, mismidad, ni lo idéntico: no hay dos iguales, "el lenguaje de la igualdad se deshace, porque uno no es ni igual ni desigual" (Contreras, 2013). ¡Cuidado!, la igualdad se volvió equivalente a homogeneidad, a lo mismo, a la identidad.

¿Por qué la lógica clasificatoria se constituye en un criterio ordenador en los programas deportivos, recreativos y de actividad física? Quizás hemos sido incapaces de pensar la diferencia en sí misma. Tal vez, hay que ampliar los rangos educativos de lo diferencial más allá de la organización de programas que clasifican y tratan a las personas como diferentes bajo criterios homogéneos, de funcionalidad y semejanzas.

Desde Aristóteles, la noción de diferencia se mantiene en el ámbito de la representación. Con Deleuze (2009), "representar la diferencia significa hacer de ella algo determinado; la diferencia puede presentarse de diferentes modos y con distintos grados de intensidad" ( $p$. 45).

Para autores de la filosofía de la diferencia como Deleuze (2009), Lyotard (1999) y Derrida (1968, 1989) la diferencia es aquello por lo cual lo dado se da como diverso, de distintas formas, permite lo múltiple, lo heterogéneo y tiene que ver con lo que nos distingue, no necesariamente de otros, porque cada sujeto es diferente en sí mismo, es singular y, en este sentido, es un modo de ser. Recordemos la noción derridiana

\section{REFERENCIAS BIBLIOGRÁFICAS}

Aldana, D.; Vargas, D.; Ramírez M.; Martínez, C.; Bohórquez, L. y Mosquera, Z. (2011). Lineamientos para la implementación del enfoque poblacional diferencial en el sector cultura, recreación y deporte, para los campos del arte, las prácticas culturales y el patrimonio. Recuperado de http:// www.culturarecreacionydeporte.gov.co/portal/ sites/default/files/Documento $\% 20$ Enfoque $\% 20$ Poblacional\%202011.pdf

Alzate, M., Fragoso, I. y Sarmiento, C. (2009). Inclusión social a través de la práctica deportiva de jóvenes en de différance, proceso dinámico de diferenciación, de producción de las diferencias individuales. La diferencia no debe ser confundida con identidad, oposición, semejanza o juicio; es un exponente del pensamiento de la alteridad y de la producción de algo nuevo.

Pensar qué hay en medio de la educación y las diferencias exige detenernos en cómo miramos, en cómo dirigimos la mirada al otro, a lo otro: miradas que inician en el otro, un otro que (nos) interroga, conmueve, afecta y desacomoda $i$ Por qué mirar un cuerpo deriva en ciertos lenguajes? Se nombran, clasifican, descalifican y rotulan los cuerpos como normales y anormales bajo una forma de ¡mirar y juzgar!; prescriptiva, regulada, representativa, modélica, vigilante, hegemónica; mirada vaga, imprecisa, distorsionada y ambigua. Se captura un cuerpo sin cuerpo, se objetualiza, pero una persona no es tan solo un brazo rígido, una pierna con prótesis, un oído sordo, un ojo ciego, una boca enmudecida, una espalda jorobada. El cuerpo es también lugar de alteridades, plural, ¡cuerpos que no solo responden a representaciones orgánicas!

Quizá mirar con otros ojos sea ponernos en el lugar de la perplejidad que nos genera un cuerpo -una persona- que tiene una lengua, un rostro, un nombre, una biografía, una emoción, una historia, una vivencia, lo cual implica acogerlo en su singularidad y rechazar cualquier normalización. Aquí hay que hacer resonar el lenguaje de la educación como una ética singular que se ocupe de la alteridad con hospitalidad, acogida, amorosidad, contacto, amistad, respeto, escucha, atención. ¡A cualquiera, todos sin excepción!, cualquier niño, cualquier joven, cualquier viejo, cualquier cuerpo...

situación de discapacidad pertenecientes al sistema de salud de las fuerzas militares de Colombia. Trabajo de especialización. Bogotá: Universidad de la Sabana.

Benjumea, M. (2011). La formación ciudadana dinamizada desde la motricidad como campo de configuración de lo humano. Un análisis desde escenarios y prácticas recreativas y deportivas. Tesis doctoral. Medellín: Universidad de Antioquia, Facultad de Educación.

Cajibioy, I. y Sevilla, E. (2013). Contexto educativo. Encuentros y desencuentros de la diversidad. Trabajo 
de Maestría. Manizales: Facultad de Ciencias Sociales y Humanas, Universidad de Manizales.

Carpintero, E. (2009). Normalidad y normalización. La salud es soporte de la anormalidad que nos hace humanos. Topía: Psicoanálisis, Sociedad y Cultura XIX(55), 3-4.

Contreras, J. (2013). Percibir la singularidad y también las posibilidades en las relaciones educativas ¿una pedagogía de la singularidad? Diplomado Pedagogías de las diferencias. Buenos Aires: Flacso Virtual.

De la Vega, E. (2010). Anormales, deficientes y especiales. Genealogía de la Educación Especial. Buenos Aires: Novedades Educativas.

Deleuze, G. (2009). Diferencia y repetición. Buenos Aires: Amorrortu.

Derrida, J. (1968). La diferencia (différance). [En línea]. Recuperado de: http://www.jacquesderrida.com.ar/ textos/la_differance.htm

Derrida, J. (1989). La escritura y la diferencia. Barcelona: Anthropos.

Dueñas, M.L. (2010). Educación inclusiva = Inclusive education. REOP-Revista Española de Orientación y Psicopedagogía 21(2), 358-366.

Duschatzky, S. y Skliar, C. (2001). Los nombres de los otros. Narrando a los otros en la cultura y en la educación. En: J. Larrosa y C. Skliar (comp.). Habitantes de babel (pp. 185-212). Barcelona: Laertes.

Dussel, I. (2013).Igualdad y diferencia en el contexto educativo. Diplomado Pedagogías de las Diferencias. Buenos Aires: Flacso Virtual.

Foucault, M. (2007). Los anormales. Buenos Aires: Fondo de Cultura Económica.

Frigerio, G. y Diker, G. (comp.) (2010). Educar: ese acto político. Buenos Aires: Fundación La Hendija.

Gordillo, M. V. (2007). Masculinidad y feminidad: cómo educar en la diferencia. Revista Española de Pedagogía LXV(238), 447-458.

Guzmán 0. (2013). Certificación de discapacidad como herramienta para la accesibilidad a derechos e inclusión social. Revista de Salud Pública 15 (1), 149157.

Hurtado, D. et al. (2005). Sistematización de experiencias deportivas y/o recreativas que en medio de contextos de violencia aportan a la construcción de tejido social y a la convivencia en Medellín. Medellín: Instituto de Deportes y Recreación de Medellín (Inder), Instituto de Estudios Políticos Universidad de Antioquia.
Irigary, H. y Vergara, S. (2011). The time as a dimension of research on diversity policy and labor relations. Cadernos EBAPE. BR 9(4), 1085-1098.

Jaramillo, E. y Mejía, M. (2012). Boccias como elemento de inclusión social. Revista Colombiana de Medicina Física y Rehabilitación 22(2), 109-116.

Krüger, H. (1999). La hermenéutica científico-espiritual. Introducción a las teorías y métodos de la ciencia de la educación. 2a. edición. Trad. Andrés Klaus Runge. Opladen: Leske y Budrich.

Loaiza, J. (2013). Análisis de la implementación de políticas públicas en el deporte en el Valle del Cauca a partir de la Constitución de 1991 al 2011. Trabajo de grado, Instituto de Educación y Pedagogía, Universidad del Valle.

Lyotard, J.F. (1999). La diferencia. Barcelona: Gedisa.

Mèlich, J.C. (2010). Ética de la compasión. Barcelona: Herder.

Pérez de Lara, N. (2009). 2010). La experiencia de la diferencia en la investigación. Investigar la experiencia educativa (pp. 117-135). Ministerio de Educación Nacional (MEN) (2011). Plan Nacional de Desarrollo 2010-2014. Prosperidad para todos.

Organización de las Naciones Unidas (ONU) (2006). Preguntas frecuentes sobre el enfoque de derechos humanos en la cooperación para el desarrollo. Oficina del Alto Comisionado de las Naciones Unidas para los Derechos Humanos. Nueva York y Ginebra.

Pérez, Á. (2013). El estilo actitudinal: una propuesta para todos y todas desde la inclusión en la educación física. Revista Lúdica Pedagógica, 2(18), 81-92.

La construcción de la identidad desde la perspectiva de la diferencia sexual. Curso Pedagogías de las Diferencias. Buenos Aires: Flacso Virtual.

Pérez de Lara, N. (En: J. Contreras y N. Pérez de Lara (comps.).Madrid: Ediciones Morata.

Pié A. (2014). Por una corporeidad postmoderna. Nuevos tránsitos sociales y educativos para la interdependencia. Barcelona: Editorial UOC.

Planella J. y Pié, A. (2012). Pedagoqueer: resistencias y subversiones educativas. Educación XXI 15(1), 265283.

Ribetto, A. (2005). Políticas de/en la diferencia y formación de profesores latinoamericanos. Conversaciones sobre el otro. Polis, 11, 1-11.

Ripamonti, P. (2011) ¿Acaso soy yo esa mano? reflexiones filosóficas en torno de la cuestión de la diferencia en contextos escolares. Revista Sul-Americana de Filosofia e Educação, 17, 78-88. 
Salomone, R. (2007). Igualdad y diferencia. La cuestión de la equidad de género en la educación. Revista Española de Pedagogía LXV(238), 433-446.

Sánchez, A., Díaz, C., Sanhueza, S. y Friz M. (2008). Percepciones y actitudes de los estudiantes de pedagogía hacia la inclusión educativa. Estudios Pedagógicos 34(2), 169-178.

Skliar, C. (2002). Alteridades y pedagogías. O... ¿y si el otro no estuviera ahí? Educação \& Sociedade XXIII(79), 85123.

Skliar, C. (2005). Poner en tela de juicio la normalidad, no la anormalidad. Políticas y falta de políticas en relación con las diferencias en educación. Revista Educación y Pedagogía XVII(41),11-22.

Skliar, C. (2008). ¿Incluir las diferencias? Sobre un problema mal planteado y una realidad. Orientación y sociedad, 8, 1-17.

Skliar, C. (2011). ¿Y si otro no estuviera ahí? Notas para una pedagogía (improbable) de la diferencia. Buenos Aires: Miño Dávila.

Skliar, C. y Bárcena, F. (2013). Cartas sobre la diferencia. Una cuestión de palabras (entre la amistad, la incomodidad y el sinsentido). Plumilla Educativa, 12, 11-28.

Skliar, C. y Larrosa, J. (comp.). (2009). Experiencia y alteridad en educación. Buenos Aires: Homo Sapiens Ediciones.

Skliar, C. y Pérez, A. (2014). Lo jurídico y lo ético en el campo de la educación: la enunciación de la diversidad y las relaciones de alteridad. Revista Temas de Educación 19(2), 9-25.
Skliar, C. y Téllez, M. (2008). Conmover la educación. Buenos Aires: Noveduc.

Sosa, L. (2007). Los "cuerpos discapacitados": construcciones en prácticas de integración en educación física. En: D. Eusse (ed.). Educación Física y discapacidad. Prácticas corporales inclusivas (pp. 93105). Medellín: Funámbulos editores, Universidad de Antioquia.

Sosa, L. (2009). Reflexiones sobre la discapacidad. Dialógica de la inclusión y exclusión en las prácticas. Ágora para la EF y el Deporte, 9, 57-82.

Vásquez, A. (2012). Representaciones sociales, inclusión de género y sexo en los juegos recreativos tradicionales de la calle de Caldas-Antioquia, Colombia. Estudios pedagógicos 38(ESPECIAL), 371-391.

Veiga-Nieto, A. (2000). Incluir y excluir. En: J. Larrosa y C. Skliar (comp.). Habitantes de babel (pp.165-184). Barcelona: Laertes.

Vélez, B. (2011). Fútbol desde la tribuna: pasiones y fantasías. Medellín: Sílaba Editores.

Yarza, A. (2007). Algunos modos de historiar la educación especial en Colombia: una mirada crítica desde la historia de la práctica pedagógica. Revista Brasileira de Educação Especial 13(2), 173-188

Yarza, A. y Rodríguez L. (2009). Educación sensorial, educación física, gimnástica y pedagogía de anormales: disciplinamiento y docilización de "corporalidades anormales" en Colombia, 1920-1940. Educación física y deporte 24(2), 11-28. 